\title{
Formal Welfarism and Intergenerational Equity
}

Cl. d'Aspremont

Discussion Paper 2005-51

Département des Sciences Économiques

de l'Université catholique de Louvain 


\section{Formal Welfarism and Intergenerational Equity* Claude d'Aspremont \\ CORE, Louvain University, Louvain-la-Neuve}

\section{Introduction}

Intergenerational justice is a matter that should primarily concern the present generation, since the individuals living now are those to take immediate decisions affecting generations that will be living in the future, and even in the far future as we know, for example, from the exhaustibility of some resources or from the long-term effects of pollution such as Global Warming. Of course, each future generation will become "present" at some point in time, and the reasoning followed for the present "present generation" about intergenerational justice could be repeated at that point in time. But, to develop this reasoning, each present generation should have a representation of future generations interests. In that respect, a simple formulation of the problem that has been extensively analyzed consists in trying to find, under equity and efficiency conditions, an ordering of the set of possible "infinite utility streams", that is, of the set of possible infinite sequences of utility levels attached to the successive generations starting with the present generation. In such a formulation, the welfare of each generation is represented by a single utility level, as if a generation were composed of a single individual or of a cohort of identical individuals with identical allocation. Even though this formulation owes so much to Ramsey (1928), the particular ranking criterion he proposed - that of maximizing the sum of undiscounted utility - was to be rejected for its limitations (see Chakravarty, 1962), and the possibility of representing an ordering of utility streams by a collective utility function (or social welfare function), treating all generations equally, was put into serious questioning. Impossibility results by Koopmans (1960), Diamond (1965) have been followed by others (e.g. Basu and Mitra, 2003a). However, if instead of looking for a social welfare function defined on infinite utility streams, one looks for a "social welfare ordering" of these streams, then, as shown non-constructively by Svensson (1980), satisfying a strong Pareto condition and treating generations equally, in some limited sense, become possible.

Our purpose, here, is neither to pursue the investigation of the general possibility or impossibility issue in this formulation of the problem of justice among generations, nor to re-examine the necessity of discounting to obtain a social welfare function. Other chapters of this Volume treat these questions. We want to look at the foundations of this formulation within the "social welfare functional" approach to social choice (as introduced by Sen, 1970). In that respect it can be seen as a supplement to the overview given in d'Aspremont and Gevers ${ }^{1}$ (2002), where the intergenerational problem is not treated.

To think about intergenerational justice in terms of infinite generational utility streams, and to look for a social welfare ordering defined on the set of such 
streams, presume a double reduction. The first is the classical "welfarist" reduction, as usually defined in ethics and social choice theory, namely that "utility" provides all the information required to construct a social evaluation rule. Of course the strenght of this reduction depends on the precise interpretation given to the concept of utility (for a discussion, see Mongin and d'Aspremont, 1998). We shall not discuss various possible interpretations here and limit our analysis to the formal consequences of welfarism, an attitude that may be called "formal welfarism." But the welfarist reduction is not the only one to be subsumed in the infinite utility stream approach. There is also, for each generation, the aggregation of the individual utility levels at each generation into a single utility level. We shall argue that this second type of reduction is not innocuous either. Not only does it require to impose additional assumptions on the social welfare functionals taken as the primitive concept for evaluating social states. It obliterates the relationship between the value judgments made in the social evaluation of the welfare of the set of individuals forming the present generation with that of future generations. To defend this argument, we rely on standard results in social choice theory showing the capacity for some social evaluation criteria to proliferate (Sen, 1977, and Hammond, 1979), in the sense that adopting such a criterion for a subgroup of individuals (e.g. the present generation) forces an ethical observer to use the same criterion for any larger group (e.g. any larger set of generations). The consequences of adopting some proliferating criterion in evaluating infinite utility streams, are better examined if these streams remain disaggregated at the individual level, allowing to apply the criterion to a subgroup of individuals, and most importantly to the present generation, and also to exploit the bulk of social choice theory as developed for the finite case. In particular, our results concerning the orderings generated respectively by the pure utilitarian rule and the Leximin rule, both having the proliferating property, are compared to the characterisations given by Basu and Mitra (2003b), Asheim and Tungodden (2004) and Bossert, Sprumont and Suzumura (2004) on different infinite-horizon extensions of these rules. To keep with the idea of an overview though, we try to be more general and derive a characterization result (the general overtaking theorem), as well as a simplified criterion, that can be associated to any rule having the proliferating property.

\section{Welfarism for successive generations}

We consider a countably infinite set of time periods, starting from the present one and denoted by $\mathbb{T}=\{0,1, \ldots, t, \ldots\}$. Associated to each time period $t$ there is a "generation" made up of a finite set $N_{t}$ of $n_{t}$ individuals and there is a set $X_{t}$ of possible social states. The set of all individuals is represented as a partition of the set of positive integers into successive generations:

$$
\mathbb{N}=\left\{N_{0}, N_{1}, \ldots, N_{t}, \ldots\right\}
$$

We assume that $n_{t} \geq 2$ and $\left|X_{t}\right| \geq 3$, for every $t$. Our objective is to evaluate the respective merits of each programme of social states $x=\left\{x^{t}\right\} \in \mathbb{X} \equiv \times_{t=1}^{\infty} X_{t}$ 
for an infinite future, taking into account individual evaluations. The final evaluation, of course, will have to take into account feasibility constraints, and in particular that the set of social states at some period may depend on the social states realised in previous periods. Here, however, we shall focus on the definition of general evaluation criteria applicable to any set of feasible programmes. Individuals are supposed to live a finite number of periods. For the present analysis we keep in mind two standard cases, the case where each individual lives only one period $\left(N_{t}\right.$ is the set of individuals living at period $t$ ), and the case where individuals live for two periods $\left(N_{t}\right.$ is the set of individuals born at period $t$ ) and generations overlap. In both cases, $N_{t}$ is the set of individuals belonging to generation $t$.

To introduce intergenerational evaluation a simple and usual approach is to suppose that, for each possible programme of social states, a "utility level" $u^{t}$ can be attached to each generation $t$, allowing to define an infinite "utility stream" $u=\left(u^{0}, u^{1}, \ldots, u^{t}, \ldots\right) \in \mathbb{R}^{\mathbb{T}}$ (with $\mathbb{R}$ the set of real numbers), and then to look for an ordering of all infinite utility streams satisfying some efficiency or equity properties. However this approach requires to proceed in two stages. The first stage is to construct an ordering of all infinite individual welfare evaluation streams (or "utility streams"). The second is to determine by aggregation, for every infinite individual welfare evaluation stream, the welfare level attached to each generation, and then to reduce the previous ordering to an ordering defined on the set of all infinite generational welfare evaluation streams.

To examine these problems we start from the concept introduced by Sen (1970) of a "Social Welfare Functional", using it both in the case of nonoverlapping and in the case of overlapping generations, and then go on to the associated concept of "Social Welfare Ordering" (the terminology fixed by Gevers, 1979).

This, formally, consists in assuming that we have an individual evaluation function (or profile for short) given by a real-valued function $U$ defined on $\mathbb{X} \times \mathbb{N}$. That is, if $i \in N_{t}$ for any $t \in \mathbb{T}$, the function $U(., i)$, or $U_{i}$ for short, is a realvalued function, defined on $X_{t} \times X_{t+1}$ in the overlapping generation case and on $X_{t}$ in the non-overlapping case, and is called individual $i$ 's evaluation function. Also, for every $x \in \mathbb{X}$, the vector $U(x,$.$) , or U_{x}$ for short, is a point in the infinite individual welfare evaluation space $\mathbb{R}^{\mathbb{N}}$ and called an infinite individual welfare evaluation stream associated to $x$. Given any individual evaluation function $U$ in some admissible subset $\mathcal{D} \subset\{U \mid U: \mathbb{X} \times \mathbb{N} \rightarrow \mathbb{R}\}$, we are to recommend a social ranking of $\mathbb{X}$, that is an element in the set $\mathcal{R}$ of all complete and transitive binary relations on $\mathbb{X}$. A social welfare functional (SWFL) is a map $F: \mathcal{D} \rightarrow \mathcal{R}$ with generic image $R_{U}=F(U)\left(I_{U}\right.$ and $P_{U}$ denoting respectively the associated indifference and strict preference relations). If $x$ is ranked socially at least as high as $y$ whenever the relevant profile is $U$, we write $x R_{U} y$ (resp. $x I_{U} y$ or $x P_{U} y$ in case of indifference or strict preference).

To reduce the SWFL approach to the comparisons of generational welfare evaluation streams, we need to introduce conditions ensuring that the relative welfare (in a formal sense) of two social states can be entirely judged by comparing their respective individual evaluation vectors, independently from the 
other aspects of the individual profile at hand. With individuals partitioned in a sequence of successive generations, formal welfarist social evaluation may be defined and applied at different levels, according to the domain of evaluation vectors which is considered (individual or generational). Standard conditions are the following:

Domain Attainability (AD). $\forall u, v, w \in \mathbb{R}^{\mathbb{N}}, \exists x, y, z \in \mathbb{X}, \exists U \in \mathcal{D}$ such that $U_{x}=u, U_{y}=v$ and $U_{z}=w$.

This condition ensures that the set $\left\{r \in \mathbb{R}^{\mathbb{N}} \mid \exists x \in \mathbb{X}, \exists U \in \mathcal{D}\right.$ such that $\left.U_{x}=r\right\}$ fills the whole individual evaluation space $\mathbb{R}^{\mathbb{N}}$. The next condition is a Paretian principle:

Pareto Indifference (PI). $\forall U \in \mathcal{D}, \forall x, y \in X$, if $U_{x}=U_{y}$ then $x I_{U} y$. The third condition is an Arrowian inter-profile consistency requirement imposing that the ranking of two alternatives depends only on the evaluation of these two alternatives.

Binary Independence (BI). $\forall U, V \in \mathcal{D}, \forall x, y \in \mathbb{X}$ such that $V_{x}=U_{x}, V_{y}=$ $U_{y}, x R_{U} y \Leftrightarrow x R_{V} y$.

As it will be recalled in the next theorem, these three conditions characterise formal welfarism, that is, the possibility to define, on the individual evaluation space $\mathbb{R}^{\mathbb{N}}$, an ordering $R^{*}$, called a Social Welfare Ordering (SWO), which is derived from the SWFL $F$. Now, since we also need to introduce the possibility of aggregating the welfare of each generation, we have to add three conditions which refer explicitly to generations. The first is a separability condition on $F$ which allows to isolate the evaluation of the welfare of each generation.

Generational Separability (GS). $\forall t \in \mathbb{T}, \forall U, V \in \mathcal{D}$, if $\forall i \in N_{t}, U_{i}=V_{i}$ whereas, $\forall j \in \mathbb{N} \backslash N_{t}, \forall x, y \in \mathbb{X}, U(x, j)=U(y, j)$ and $V(x, j)=V(y, j)$, then $R_{U}=R_{V}$.

This condition allows to derive from $F$, for each generation $t$, a SWFL $F_{t}$ defined on a domain $\mathcal{D}_{t}$ contained in $\left\{U^{t} \mid U^{t}: \mathbb{X} \times N_{t} \rightarrow \mathbb{R}\right\}$ with range in $\mathcal{R}$. For each $i \in N_{t}$, we define $U^{t}(., i)$ on the whole set $\mathbb{X}$ for simplicity of notation, but it is constant for variables outside $X_{t} \times X_{t+1}$ in the overlapping generation case and outside $X_{t}$ in the non-overlapping case. If the relevant profile is $U^{t}$ and $x \in \mathbb{X}$ is ranked socially at least as high as $y \in \mathbb{X}$, we then write $x R_{U^{t}}^{t} y\left(x I_{U^{t}}^{t} y\right.$ or $x P_{U^{t}}^{t} y$ in case of indifference or strict preference). Moreover, our first three conditions can be straightforwardly reformulated for each $t$ to be applied to each derived SWFL $F_{t}$ : replacing $\mathbb{R}^{\mathbb{N}}, \mathcal{D}, U, V, R_{U}, R_{V}$ and $I_{U}$ by, respectively $\mathbb{R}^{N_{t}}, \mathcal{D}_{t}, U^{t}$, $V^{t}, R_{U^{t}}^{t}, R_{V^{t}}^{t}$ and $I_{U^{t}}^{t}$ in the conditions $\mathrm{AD}, \mathrm{PI}$ and $\mathrm{BI}$, we get the conditions $\mathrm{AD}_{t}, \mathrm{PI}_{t}$ and $\mathrm{BI}_{t}$. They will ensure the existence of an associated SWO $R_{t}^{*}$ defined on $\mathbb{R}^{N_{t}}$.

Lemma 1 Assume the SWFL F satisfies conditions AD, PI, BI and GS. Then, for every generation $t \in \mathbb{T}$, there is a SWFL $F_{t}: \mathcal{D}_{t} \rightarrow \mathcal{R}, \mathcal{D}_{t} \subset\left\{U^{t} \mid U^{t}\right.$ : $\left.\mathbb{X} \times N_{t} \rightarrow \mathbb{R}\right\}$, which can be identified to the restriction of the $S W F L F$ to some subset $\overline{\mathcal{D}}_{t} \subset \mathcal{D}$. The $S W F L F_{t}$ satisfies the conditions $A D_{t}, P I_{t}$ and $B I_{t}$.

Proof. Under GS, $F_{t}$ can be identified to the restriction of $F$ to the set $\overline{\mathcal{D}}_{t}=\left\{U \in \mathcal{D}: \forall j \in \mathbb{N} \backslash N_{t}, \forall x \in \mathbb{X}, U(x, j)=\bar{U}(x, j)\right\}$, for some arbitrary $\bar{U} \in$ 
$\mathcal{D}$ such that $\forall x \in \mathbb{X}, \bar{U}_{x}=\bar{u} \in \mathbb{R}^{\mathbb{N}}$, so that $\mathcal{D}_{t}=\left\{U^{t} \mid U^{t}: \mathbb{X} \times N_{t} \rightarrow \mathbb{R}\right.$ and $\left.\left(U^{t}, \bar{U}^{-t}\right) \in \overline{\mathcal{D}}_{t}\right\}$, with $\bar{U}^{-t}=\left(\bar{U}_{i}\right)_{i \in \mathbb{N} \backslash N_{t}}$. Since $F$ satisfies AD, PI and BI, $F_{t}$ satisfies $\mathrm{AD}_{t}, \mathrm{PI}_{t}$ and $\mathrm{BI}_{t}$. Indeed $\mathrm{PI}$ and $\mathrm{BI}$ should hold on $\overline{\mathcal{D}}_{t}$, meaning that $\mathrm{SP}_{t}$ and $\mathrm{BI}_{t}$ hold on $\mathcal{D}_{t}$. Similarly, $\mathrm{AD}_{t}$ is simply the condition $\mathrm{AD}$ applied to all $u, v, w \in \mathbb{R}^{N_{t}} \times \mathbb{R}^{\mathbb{N} \backslash N_{t}}$ such that $u^{-t}=v^{-t}=w^{-t}=\bar{u}^{-t} \in \mathbb{R}^{\mathbb{N} \backslash N_{t}}$.

Two additional conditions, both based on the derived SWFLs $\left\{F_{t}\right\}$, are needed in the following theorem. One is continuity. We say that a sequence $\left(U^{t k}\right)_{k=1}^{\infty} \subset \mathcal{D}_{t}$ converges pointwise to $U^{t 0} \in \mathcal{D}_{t}$, if $\lim _{k \rightarrow \infty} U^{t k}(x, i)=U^{t 0}(x, i)$, $\forall(x, i) \in \mathbb{X} \times N_{t}$. The condition is then stated as

Generational Continuity $(G C) . \forall t \in N, \forall x, y \in \mathbb{X}, \forall U^{t 0} \in \mathcal{D}_{t}$ and for any sequence $\left(U^{t k}\right)_{k=1}^{\infty} \subset \mathcal{D}_{t}$ converging pointwise to $U^{t 0}$, if $x R_{U^{t k}}^{t} y, \forall k \geq 1$, then $x R_{U^{t 0}}^{t} y$.

This property will allow to represent each SWO $R_{t}^{*}$ by a continuous function $w_{t}$, called a Social Welfare Function (SWF): $\forall u^{t}, v^{t} \in \mathbb{R}^{N_{t}}, w_{t}\left(u^{t}\right) \geq w_{t}\left(v^{t}\right) \Longleftrightarrow$ $u^{t} R_{t}^{*} v^{t}$.

The last condition is an "extended Pareto" condition (Dhillon (1998)). It is a Pareto indifference condition but applied to a partition of all individuals into groups (here the generations): if all the groups are indifferent between two alternatives, then society should also be indifferent.

Generational Pareto Indifference $(G P I) . \forall\left(U^{t}\right)_{t \in \mathbb{T}} \in \times_{t \in \mathbb{T}} \mathcal{D}_{t}, \forall x, y \in \mathbb{X}$, if , $x I_{U^{t}}^{t} y, \forall t \in \mathbb{T}$, then $x I_{U} y$ with $U \in \mathcal{D}$ such that, $\forall t \in \mathbb{T}, \forall i \in N_{t}, U_{i}=U_{i}^{t}$.

We can now prove the following result.

Theorem 1 (intergenerational welfarism) Assume the SWFL F satisfies conditions $A D, S P$ and BI. Then, (i) there exists a $S W O R^{*}$ on $\mathbb{R}^{\mathbb{N}}$ such that, for all $x, y \in \mathbb{X}$ and for all $U \in \mathcal{D}$,

$$
U_{x} R^{*} U_{y} \Longleftrightarrow x R_{U} y ;
$$

also, (ii) under $G S$ and for every generation $t \in \mathbb{T}$, there exists a $S W O R_{t}^{*}$ on $\mathbb{R}^{N_{t}}$ such that, for all $x, y \in \mathbb{X}$ and for all $U^{t} \in \mathcal{D}_{t}$,

$$
U_{x}^{t} R_{t}^{*} U_{y}^{t} \Longleftrightarrow x R_{U^{t}}^{t} y ;
$$

and, (iii), with $G C$ and GPI in addition, there is, for every generation $t \in$ $\mathbb{T}$, a social welfare function $w_{t}: \mathbb{R}^{N_{t}} \longrightarrow \mathbb{R}$ such that, for all $u^{t} \in \mathbb{R}^{N_{t}}$, $\mathbf{1}_{N_{t}} w_{t}\left(u^{t}\right) I_{t}^{*} u^{t}$ (with $\mathbf{1}_{N_{t}}=(1, \ldots, 1) \in \mathbb{R}^{N_{t}}$ ) and there exists a $S W O R^{\#}$ on $\mathbb{R}^{\mathbb{T}}$ such that, for all $u, v \in \mathbb{R}^{\mathbb{N}}$,

$$
\left(w_{0}\left(u^{0}\right), w_{1}\left(u^{1}\right) \ldots, w_{t}\left(u^{t}\right), \ldots\right) R^{\#}\left(w_{0}\left(v^{0}\right), w_{1}\left(v^{1}\right) \ldots, w_{t}\left(v^{t}\right), \ldots\right) \Longleftrightarrow u R^{*} v .
$$

Proof. As is well-known from the case of a finite number of individuals, under $\mathrm{AD}$, the conditions PI and BI are equivalent to the condition of Strong Neutrality $(\mathrm{SN}): \forall U, V \in \mathcal{D}, \forall x, y \in \mathbb{X}$, if there are $x^{\prime}, y^{\prime} \in \mathbb{X}$ such that $V_{x^{\prime}}=U_{x}, V_{y^{\prime}}=U_{y}$ then $x R_{U} y \Leftrightarrow x^{\prime} R_{V} y^{\prime}$. The argument consists in choosing $z \in \mathbb{X} \backslash\left\{y, y^{\prime}\right\}$, with $z$ a third alternative if the two pairs coincide, and, thanks to AD, in 
constructing profiles $U^{1}, U^{2}$ and $U^{3}$ such that $U_{x}^{1}=U_{z}^{1}=u, U_{y}^{1}=v, U_{z}^{2}=u$, $U_{y}^{2}=U_{y^{\prime}}^{2}=v, U_{x^{\prime}}^{3}=U_{z}^{3}=u$ and $U_{y^{\prime}}^{3}=v$. Applying alternately BI and PI, we get $x R_{U^{\prime}} y \Longleftrightarrow x R_{U^{1}} y \Longleftrightarrow z R_{U^{1}} y \Longleftrightarrow z R_{U^{2}} y \Longleftrightarrow z R_{U^{2}} y^{\prime} \Longleftrightarrow z R_{U^{3}} y^{\prime} \Longleftrightarrow$ $x^{\prime} R_{U^{3}} y^{\prime} \Longleftrightarrow x^{\prime} R_{V} y^{\prime}$, and SN follows. Then, defining $R^{*}$ by (2.1), for some $x, y \in \mathbb{X}$ and for some $U \in \mathcal{D}$, we get by SN the same relation for any $x^{\prime}, y^{\prime} \in \mathbb{X}$ such that $V_{x^{\prime}}=U_{x}, V_{y^{\prime}}=U_{y}$, so that $R^{*}$ is well-defined. Completeness and transitivity of $R^{*}$ follow from $\mathrm{AD}$ and from the completeness and transitivity of each $R_{U}$. This proves (i). To prove (ii), we know from lemma 1 that each $F_{t}$ satisfies $\mathrm{AD}_{t}, \mathrm{PI}_{t}$ and $\mathrm{BI}_{t}$. Then, repeating the same argument as in (i), we get the required SWO $R_{t}^{*}$ defined on $\mathbb{R}^{N_{t}}$ (see 2.2 ). To prove (iii), we need in addition $R_{t}^{*}$ to be continuous, i.e. $\forall v^{t} \in \mathbb{R}^{N_{t}}$, that the sets $\left\{u^{t} \in R^{N_{t}} \mid u^{t} R_{t}^{*} v^{t}\right\}$ and $\left\{u^{t} \in R^{N} \mid v^{t} R_{t}^{*} u^{t}\right\}$ be closed in $R^{N_{t}}$. This property is implied by GC. Indeed, if for some $v_{t} \in \mathbb{R}^{N_{t}}$ the set $\left\{u^{t} \in R^{N_{t}} \mid u^{t} R_{t}^{*} v^{t}\right\}$, say, was not closed, it would be possible to find a sequence $\left(u^{t k}\right)_{k=1}^{\infty}$ in $\mathbb{R}^{N_{t}}$, converging to some $u^{t 0}$, with $u^{t k} R_{t}^{*} v^{t}$ for all $k \geq 1$ and $v^{t} P^{*} u^{t 0}$; but it would then be possible to construct a sequence $\left(U^{t k}\right)_{k=1}^{\infty}$ converging pointwise to $U^{t 0}$ such that, $\forall k \geq 1$, $\left(U^{t k}(x, i)\right)_{i \in N_{t}}=u^{t k}$ and $\left(U^{t k}(y, i)\right)_{i \in N_{t}}=v^{t}$ for some $x, y \in \mathbb{X}$, implying $x R_{U^{t k}} y, \forall k \geq 1$, but $y P_{U^{t 0}} x$, in contradiction with GC. Thus, we obtain (see e.g. Theorem 3 in Blackorby, Bossert and Donaldson, 2002) that, for every $t \in \mathbb{T}$, there exists a SWF $w_{t}: \mathbb{R}^{N_{t}} \rightarrow \mathbb{R}$ such that for every $u^{t} \in \mathbb{R}^{N_{t}}, \mathbf{1}_{N_{t}} w_{t}\left(u^{t}\right) I_{t}^{*} u^{t}$. Now, by $\mathrm{AD}$, for any $u \in \mathbb{R}^{\mathbb{N}}$, there are $x, y \in \mathbb{X}$ and $U \in \mathcal{D}$ such that $U_{x}=u$ and $U_{y}=\left(\mathbf{1}_{N_{0}} w_{0}\left(u^{0}\right), \mathbf{1}_{N_{1}} w_{1}\left(u^{1}\right), \ldots, \mathbf{1}_{N_{t}} w_{t}\left(u^{t}\right), \ldots\right)$ so that, by GPI, if , $x I_{U^{t}}^{t} y$, $\forall t \in \mathbb{T}$, then

$$
u I^{*}\left(\mathbf{1}_{N_{0}} w_{0}\left(u^{0}\right), \mathbf{1}_{N_{1}} w_{1}\left(u^{1}\right), \ldots, \mathbf{1}_{N_{t}} w_{t}\left(u^{t}\right), \ldots\right)
$$

So, whenever $u R^{*} v$ we may write as well,

$$
\left(\mathbf{1}_{N_{t}} w_{t}\left(u^{t}\right)\right)_{t \in \mathbb{T}} R^{*}\left(\mathbf{1}_{N_{t}} w_{t}\left(v^{t}\right)\right)_{t \in \mathbb{T}}
$$

or taking only one representative component per generation we can write equivalently

$$
\left(w_{0}\left(u^{0}\right), w_{1}\left(u^{1}\right), \ldots, w_{t}\left(u^{t}\right), \ldots\right) R^{\#}\left(w_{0}\left(v^{0}\right), w_{1}\left(v^{1}\right), \ldots, w_{t}\left(v^{t}\right), \ldots\right),
$$

thereby defining a SWO $R^{\#}$ on $\mathbb{R}^{\mathbb{T}}$.

This "welfarism theorem", as any other welfarism theorem ${ }^{2}$, opens the possibility to work directly in terms of SWOs and to add conditions formulated in those terms only. Of course these additional properties will, almost always, be easily translated back in SWFL terms.

\section{Intergenerational Social Welfare Orderings}

In the previous section we have shown that, even with an infinite number of individuals partitioned into a sequence of generations, it is possible to prove a welfarism theorem, transforming the problem of finding an acceptable Social 
Welfare Functional into the problem of finding an appropriate Social Welfare Ordering. The theorem above even left us with two possible SWOs: $R^{*}$ or $R^{\#}$. Formally they are completely similar. Both are orderings of all infinite evaluation streams and, if we want to get a SWO satisfying both collective efficiency and intergenerational equity conditions, the problem of constructing the ordering remains as difficult whether in the case of $R^{*}$ or in the case of $R^{\#}$. Existence of such an ordering is difficult to establish. We shall rely on the result by Svensson (1980). Other existence results are provided in Fleurbaey and Michel (2003). In that respect, the literature studies principally the SWO $R^{\#}$. We want to argue that it is preferable to work with the more basic SWO $R^{*}$. A first advantage is that $R^{*}$ can be derived from a SWFL under a weaker set of assumptions (using neither GS nor GC nor GPI). However, this is a formal argument and, for some results, we will have to add separability or continuity assumptions anyway. The main argument in favour of $R^{*}$ is that it forces to consider explicitly the relationship between the problem of justice among generations and the problem of justice among individuals within each generation and, primarily, within the present generation. Shouldn't the solutions proposed for the intra-generational problem, which has up to now been the main domain of investigation in ethics and social choice, have some bearing on the solutions that should be considered for the inter-generational problem? Using standard welfarist arguments, this section answers positively to this question.

To represent collective efficiency and intergenerational equity requirements, the two basic conditions that we shall use are the following. The first is the welfarist translation of the strong Paretian condition, Pareto indifference being trivially satisfied by construction of $R^{*}$ :

Strong Pareto $\left(S P^{*}\right) . \forall u, v \in R^{\mathbb{N}}$, if $u \geq v$ and $u \neq v$, then $u P^{*} v$.

By $u \geq v$ we mean $u_{i} \geq v_{i}, \forall i \in \mathbb{N}$. This is a strong but standard efficiency condition.

As for equity, the basic condition is to keep social indifference for finite permutations of individual evaluations both within and across generations. Although this condition seems to be introducing a minimal condition of impartiality, it already excludes the use of a discount factor. A much more demanding condition would be to allow for all permutations, but then it becomes incompatible with Pareto conditions (see Lauwers and Van Liedekerke, 1995, Lauwers, 1998). Other, intermediate, impartiality conditions are studied in Fleurbaey and Michel (2003).

Finite Anonymity $\left(F A^{*}\right)$. If $\sigma$ is a permutation of $M \subset \mathbb{N},|M|<\infty$, and $u, v \in \mathbb{R}^{\mathbb{N}}$ are such that $u_{i}=v_{i}, \forall i \in \mathbb{N} \backslash M$, and $u_{i}=v_{\sigma(i)}, \forall i \in M$, then $u I^{*} v$.

The two conditions $\mathrm{SP}^{*}$ and $\mathrm{FA}^{*}$ are defined as properties of a SWO $R^{*}$ defined in $\mathbb{R}^{\mathbb{N}}$. But they can also be defined as properties of a quasi-ordering $R$, that is, a reflexive and transitive binary relation defined in $\mathbb{R}^{\mathbb{N}}$ (resp. in a suspace $\mathbb{R}^{M}, M \subset \mathbb{N}$, or just in $\mathbb{R}^{m}, m<\infty$, with $\mathrm{SP}^{*}$ and $\mathrm{FA}^{*}$ then restricted to such domains), which we call a Social Welfare Quasi-ordering (SWQ) on $\mathbb{R}^{\mathbb{N}}$ (resp. on $\mathbb{R}^{M}$ or on $\mathbb{R}^{m}$ ). A SWO is a complete SWQ. A SWQ $R$ is a sub-relation to another SWQ $R^{\prime}$ (or, equivalently, $R^{\prime}$ is an extension of $R$ ) if they have the same domain and for any $u$ and $v$ in this domain, $u P v \Longrightarrow u P^{\prime} v$ and $u I v \Longrightarrow u I^{\prime} v$ 
( $P$ and $I$ denoting respectively the strict preference and indifference relations associated to $R$ ). If $R^{\prime}$ is a SWO, then $R^{\prime}$ is called an ordering extension of $R$ (see Bossert, Sprumont and Suzumura, 2004).

Combining the two basic conditions we obtain a well-known SWQ, first proposed by Suppes (1966) and further analysed by Sen (1970), Kolm (1972) and Hammond $(1976,1979)$. This version is adapted to the infinite case (see Svensson, 1980).

Definition 1 (The $\mathbf{m}$-Grading Principle) The m-Grading Principle is the $S W Q R^{S}$ such that: for any permutation $\sigma: M \rightarrow M, M \subset \mathbb{N}$ with $|M|=m$, and for any $u, v \in R^{\mathbb{N}}$, if $u_{i} \geq v_{\sigma(i)}, \forall i \in M$ and $u_{i} \geq v_{i}, \forall i \in \mathbb{N} \backslash M$, then $u R^{S} v$; if in addition $u_{j}>v_{\sigma(j)}$ for some $j \in M$, then $u P^{S} v$.

The $m$-Grading Principle is a quasi-ordering on $\mathbb{R}^{\mathbb{N}}$ that satisfies both $\mathrm{SP}^{*}$ and FA*. The following result, easily adapted from lemma 3.1.1 in d'Aspremont (1985), demonstrates the capacity (at least known ${ }^{3}$ since Sen, 1977) of the Grading Principle to proliferate through and across generations:

Lemma 2 If a $S W Q R$ is an extension of the 2-Grading Principle then it satisfies $S P^{*}$ and $F A^{*}$. Moreover, if $R$ satifies these two conditions, then $R$ is an extension of the $m$-Grading Principle for every $m<\infty$.

Proof. The only new argument (with respect to the finite case) is to show that the 2-Grading Principle implies SP*. But, for $u, v \in R^{\mathbb{N}}$, supposing $u \geq v$ and $u_{j}>v_{j}$ for some $j$, and applying the 2-Grading Principle to the pair $\{j, j+1\}$, we immediately get $u P^{*} v$.

There are, of course, many quasi-orderings satisfying $\mathrm{SP}^{*}$ and $\mathrm{FA}^{*}$, but all have the $m$-Grading Principle as sub-relation. The interest in the Grading Principle in comparing infinite utility streams comes from the following existence theorem for SWOs given by Svensson (1980, theorem 2), and based on a result due to Szpilrajn (1930) and adapted by Arrow (1951, section 3 of chapter VI).

Theorem 2 (Svensson, 1980) If a $S W Q R$ is an extension of the $m$-Grading Principle for every $m<\infty$, then there exists a $S W O R^{*}$ which is an ordering extension of $R$ (and hence $R^{*}$ satisfies $S P^{*}$ and $F A^{*}$ ).

An important observation is that the "proliferating" property of a SWQ, as illustrated by the Grading Principle, can be defined in general terms.

Definition 2 (proliferating sequence) For any $M \subset \mathbb{N},|M|=m, 2 \leq m<$ $\infty$, let $R_{m}$ denote a $S W Q$ defined on $\mathbb{R}^{m}$ ( with $P_{m}$ and $I_{m}$ denoting respectively the associated strict preference and indifference relations) and, for any $u \in \mathbb{R}^{\mathbb{N}}$, let $u_{M} \in \mathbb{R}^{m}$ be such that $u_{M}=\left(u_{i}\right)_{i \in M}$. Then:

(i) A $S W Q R$ is said to extend $R_{m}$ if, $\forall M \subset \mathbb{N},|M|=m, \forall u, v \in \mathbb{R}^{\mathbb{N}}$, if $u_{M} P_{m} v_{M}$ and $u_{j} \geq v_{j}$ (resp. $u_{M} I_{m} v_{M}$ and $\left.u_{j}=v_{j}\right), \forall j \in \mathbb{N} \backslash M$, then $u P v$ (resp. uIv).

(ii) A sequence of $S W Q s\left(R_{m}\right)_{m=2}^{\infty}$, with each $R_{m}$ defined on $\mathbb{R}^{m}$, is said to be proliferating if every $S W Q R$ defined on $\mathbb{R}^{\mathbb{N}}$ and extending $R_{2}$, also extends $R_{m}$ for every $m<\infty$. 
A useful property of a proliferating sequence is the following:

Lemma 3 Consider a proliferating sequence of $S W Q s\left(R_{m}\right)_{m=2}^{\infty}$, with each $R_{m}$ defined on $\mathbb{R}^{m}$. If a $S W Q R$ defined on $\mathbb{R}^{\mathbb{N}}$ extends $R_{2}$ and $R_{2}$ satisfies $S P^{*}$ and $F A^{*}$ restricted to $\mathbb{R}^{2}$, then $R$ satisfies $S P^{*}$ and $F A^{*}$.

Proof. Since $R_{2}$ satisfies $\mathrm{SP}^{*}$ and $\mathrm{FA}^{*}$ restricted to $\mathbb{R}^{2}$, it is an extension of the 2-Grading Principle on $\mathbb{R}^{2}$ (see, e.g., lemma 3.1.1 in d'Aspremont (1985)). So $R$ is an extension of the 2-Grading Principle on $\mathbb{R}^{\mathbb{N}}$ and the result follows from Lemma 2.

Clearly, by Lemma 2, the sequence of SWQs corresponding to the $m$-Grading Principle (as defined on $\mathbb{R}^{m}$ ) is proliferating. But there are other well-known proliferating sequences. One example is based on the pure utilitarian rule.

Definition 3 (Pure $m$-Utilitarianism) The pure utilitarian $S W O$ on $\mathbb{R}^{m}$, denoted $R_{m}^{p u}$, is such that: for any $u, v \in \mathbb{R}^{m}, u R_{m}^{p u} v$ if only if $\sum_{i=1}^{m} u_{i} \geq$ $\sum_{i=1}^{m} v_{i}$.

We call $\left(R_{m}^{p u}\right)_{m=2}^{\infty}$ the pure utilitarian sequence. We then have:

Lemma 4 The pure utilitarian sequence $\left(R_{m}^{p u}\right)_{m=2}^{\infty}$ is proliferating.

Proof. The proof (adapted from lemma 3.3.1 in d'Aspremont, 1985) goes by induction. Suppose a SWQ $R$ extends $R_{2}^{p u}, R_{3}^{p u}, \ldots$, and $R_{m}^{p u}$, we want to show that it extends $R_{m+1}^{p u}$. Take any $M \subset \mathbb{N},|M|=m \geq 2$, any $j \in \mathbb{N} \backslash M$, and any $u, v \in \mathbb{R}^{\mathbb{N}}$ such that $u_{i} \geq v_{i}, \forall i \in \mathbb{N} \backslash M^{\prime}$, with $M^{\prime}=M \cup\{j\}$. For simplicity of notation, suppose $M^{\prime}=\{1,2, \ldots, m\} \cup\{m+1\}$. Then, we can find $w \in \mathbb{R}^{\mathbb{N}}$ such that $w_{i}=u_{i}$ for $1 \neq i \neq m+1$,

$$
w_{1}+w_{m+1}=u_{1}+u_{m+1},
$$

and $w_{m+1}=v_{m+1}$. Since $R$ extends $R_{2}^{p u}$, we have $w I u$. Also, since $R$ extends $R_{m}^{p u}$, we get

$$
\sum_{i=1}^{m+1} u_{i}=\sum_{i=1}^{m+1} w_{i}>\sum_{i=1}^{m+1} v_{i} \Longrightarrow \sum_{i=1}^{m} w_{i}>\sum_{i=1}^{m} v_{i} \Longrightarrow w P v \Longrightarrow u P v
$$

and $u I v$ if the inequalities are replaced by equalities.

Hence, if the pure utilitarian rule is used to evaluate the welfare of a set of individuals belonging to some generation, say the present generation, then it has to be used to evaluate the welfare of any finite set of subsequent generations.

A second example is the Leximin (the lexicographic completion of the maximin), the proliferating property of which is known since Sen $(1976,1977)$ and Hammond (1979). We start by defining $m$-Leximin using, for any $u \in \mathbb{R}^{m}$, the notation $u_{i(.)} \in \mathbb{R}^{m}$ to denote the vector with the same set of components as $u$ but increasingly ranked.

Definition 4 ( $m$-Leximin) For $2 \leq m<\infty$, the $m$-Leximin $S W O$ on $\mathbb{R}^{m}$, denoted $R_{m}^{l x}$, is such that: for any $u, v \in \mathbb{R}^{m}, u P_{m}^{l x} v$ if and only if $\exists k \in\{1,2, \ldots, m\}$ such that $u_{i(k)}>v_{i(k)}$ and $u_{i(h)}=v_{i(h)}$, for $h=1,2, \ldots, k-1$. 
We call $\left(R_{m}^{l x}\right)_{m=2}^{\infty}$ the Leximin sequence. The proliferating property of $m$ Leximin can be shown by a simple adaptation of the argument in d'Aspremont (1985, lemma 3.4.1).

Lemma 5 The Leximin sequence $\left(R_{m}^{l x}\right)_{m=2}^{\infty}$ is proliferating.

Proof. The proof goes by induction. But, first, it can be verified that if a SWQ $R$ extends $R_{2}^{l x}$, then $R$ is an extension of the 2-Grading Principle, hence, by lemma $2, R$ satisfies $\mathrm{SP}^{*}$ and FA* Suppose now that $R$ extends $R_{2}^{l x}, R_{3}^{l x}, \ldots$, and $R_{m}^{l x}$. We want to show that it extends $R_{m+1}^{l x}$. With FA* we can take $M=\{1,2, \ldots, m\}$, and $m+1 \in \mathbb{N} \backslash M$, and consider any $u, v \in \mathbb{R}^{\mathbb{N}}$ such that $u_{i} \geq v_{i}, \forall i>m+1, u_{1} \leq u_{2} \leq \ldots \leq u_{m+1}$ and $v_{1} \leq v_{2} \leq \ldots \leq v_{m+1}$. Clearly $u I v$ if and only if $u=v$. If $u_{i}>v_{i}$, for some $i>1$, and $u_{j} \geq v_{j}$, for $j=1, \ldots, i-1$, then $u P v$ by applying $R_{k}^{l x}, 2 \leq k \leq m$, or simply by SP*. It remains to show that $u P v$, whenever $u_{1}>v_{1}$ and $u_{j}<v_{j}$, for $j=2, \ldots, m+1$, so that $v_{2}>v_{1}$ (otherwise we would have $u_{2}>v_{2}$ ). But, then, we can find $w \in \mathbb{R}^{\mathbb{N}}$ such that $v_{1}<w_{1}<\min \left\{v_{2}, u_{1}\right\}, w_{2}=u_{2}$ and $w_{h}=v_{h}, 1 \neq h \neq 2$, implying $u P w$ by application of $R_{m}^{l x}$, and $w P v$ by application of $R_{2}^{l x}$.

Our purpose in defining proliferating sequences is to apply Theorem 2 and to look for SWOs that are extensions of rules that are well-known and well characterized in the intra-generational case, such as Pure Utilitarianism and Leximin, in order to rank infinite utility streams. Indeed, the choice of such a rule for any generation constrains the choice of a similar rule for intergenerational comparisons. For that purpose we define a notion of "generalised overtaking criterion", inspired by v.Weizsäcker (1965) and Atsumi (1965), but applied to other rules than the pure utilitarian rule and adapted to the case where the individual evaluation of each generation is not aggregated. Such criteria consist in "transforming the comparison of any two infinite utility paths to an infinite number of comparisons of utility paths each containing a finite number of generations" (Asheim and Tungodden, 2004). However, the present formulation of the criterion differs in two different important respects. The first is that it aims at an ordering of all infinite individual welfare evaluation streams without aggregating them into streams of each generation utility. The second is that the criterion is not only applied to any finite number of successive generations, from some point on, but to any finite set of individuals (belonging to any generation), from some size on. This leads to a more demanding criterion (implying less completeness), but which does not privilege in a specific way the present generation and the generations in the near future. The criterion treats all generations symmetrically.

Criterion 1 (general overtaking) A SWQ $R^{o}$ defined in $\mathbb{R}^{\mathbb{N}}$ is a generalised overtaking criterion generated by a proliferating sequence of SWQs $\left(R_{m}\right)_{m=2}^{\infty}$ if it extends $R_{2}$ and is such that: $\forall u, v \in \mathbb{R}^{\mathbb{N}}, u P^{o} v$ (resp. $u I^{o} v$ ) if and only if $\exists \bar{m} \geq 2$ such that, $\forall M \subset \mathbb{N}$ with $|M|=m \geq \bar{m}, u_{M} P_{m} v_{M}$ (resp. $u_{M} I_{m} v_{M}$ ).

The proliferating feature of an overtaking sequence of SWOs implies that, accepting only the first element of the sequence, because we take it as an acceptable condition, we are forced to accept any subsequent element of the sequence. 
Hence the properties of $R_{2}$, the first accepted SWQ, are crucial. Coming back to Svensson's theorem this remark has the following important application:

Theorem 3 (general overtaking) Suppose $R^{o}$ is a generalised overtaking criterion generated by a proliferating sequence of $S W Q s\left(R_{m}\right)_{m=2}^{\infty}$, with $R_{2}$ satisfying $S P^{*}$ and $F A^{*}$ restricted to $\mathbb{R}^{2}$. Then there exists a $S W O R^{*}$ defined on $\mathbb{R}^{\mathbb{N}}$, an ordering extension of $R^{o}$, satisfying $S P^{*}$ and $F A^{*}$. Moreover, a $S W Q R$ defined on $\mathbb{R}^{\mathbb{N}}$ extends $R_{2}$ (and hence satisfies $S P^{*}$ and $F A^{*}$ ) if and only if $R^{o}$ is a subrelation of $R$.

Proof. Consider the generalised overtaking criterion $R^{o}$ generated by the sequence $\left(R_{m}\right)_{m=2}^{\infty}$. Because the sequence is proliferating, $R^{o}$ extends $R_{m}, \forall m \geq$ 2. Since $R_{2}$ satisfies $\mathrm{SP}^{*}$ and $\mathrm{FA}^{*}$ restricted to $\mathbb{R}^{2}, R^{o}$ satisfies $\mathrm{SP}^{*}$ and $\mathrm{FA}^{*}$ and is an extension of the $m$-Grading Principle for every $m<\infty$ (by lemma 2 and 3). Then, by theorem 2, there exists a SWO $R^{*}$ defined on $\mathbb{R}^{\mathbb{N}}$ which is an ordering extension of $R^{o}$ and satisfying $\mathrm{SP}^{*}$ and $\mathrm{FA}^{*}$.

Now, consider a SWQ $R$ defined on $\mathbb{R}^{\mathbb{N}}$ extending $R_{2}$, and so satisfying $\mathrm{SP}^{*}$ and $\mathrm{FA}^{*}$. Since the sequence $\left(R_{m}\right)_{m=2}^{\infty}$ is proliferating, $R$ extends all subsequent $R_{m}, 2 \leq m<\infty$. Suppose $R^{o}$, the overtaking criterion generated by this sequence, is not a subrelation of $R$. Then $\exists u, v \in \mathbb{R}^{\mathbb{N}}$ such that $u P v$ (resp. $u I v$ ) does not hold although $u P^{o} v$ (resp. $u I^{o} v$ ) holds, meaning that for some $\bar{m} \geq 2, \forall M \subset \mathbb{N},|M|=m \geq \bar{m}, u_{M} P_{m} v_{M}$ (resp. $u_{M} I_{m} v_{M}$ ). Thus, $\forall M \subset \mathbb{N},|M| \geq \bar{m}, \exists i \in \mathbb{N} \backslash M$ such that $v_{i}>u_{i}$ (resp. $v_{i} \neq u_{i}$ ). Otherwise there would be some $M \subset \mathbb{N},|M|=m \geq \bar{m}$, such that $u_{M} P_{m} v_{M}$ (resp. $u_{M} I_{m} v_{M}$ ) and, $\forall i \in \mathbb{N} \backslash M, u_{i} \geq v_{i}$ (resp. $u_{i}=v_{i}$ ) implying $u P v$ (resp. $u I v$ ), since $R$ extends $R_{m}$. So we may select sets $M_{1}, \ldots, M_{\bar{m}}$ and $M^{\prime}=\left\{i_{1}, \ldots, i_{\bar{m}}\right\}$ such that $\left|M_{k}\right| \geq \bar{m}, i_{k} \in \mathbb{N} \backslash M_{k}$, and $v_{i_{k}}>u_{i_{k}}$ for $k=1, \ldots, \bar{m}$ (resp. either $v_{i_{k}}>u_{i_{k}}$ for all $k$, or $u_{i_{k}}>v_{i_{k}}$ for all $k, 1 \leq k \leq \bar{m}$ ) and $\left\{i_{1}, \ldots, i_{k-1}\right\} \subset M_{k}$, for $k=2, \ldots, \bar{m}$. Then by $\mathrm{SP}^{*},\left(v_{M^{\prime}}, v_{\mathbb{N} \backslash M^{\prime}}\right) P\left(u_{M^{\prime}}, v_{\mathbb{N} \backslash M^{\prime}}\right)$ (resp. $\left(v_{M^{\prime}}, v_{\mathbb{N} \backslash M^{\prime}}\right) P\left(u_{M^{\prime}}, v_{\mathbb{N} \backslash M^{\prime}}\right)$ or $\left.\left(u_{M^{\prime}}, v_{\mathbb{N} \backslash M^{\prime}}\right) P\left(v_{M^{\prime}}, v_{\mathbb{N} \backslash M^{\prime}}\right)\right)$ which contradicts $u_{M^{\prime}} P_{\bar{m}} v_{M^{\prime}}\left(\right.$ resp. $\left.u_{M^{\prime}} I_{\bar{m}} v_{M^{\prime}}\right)$, since $R$ satisfies $R_{\bar{m}}$.

This theorem can be applied to any generalised overtaking criterion $R^{o}$ generated by a proliferating sequence of SWQs, whenever the first element in the sequence, $R_{2}$, satisfies $\mathrm{SP}^{*}$ and $\mathrm{FA} *$ restricted to $\mathbb{R}^{2}$. It can in particular be applied to the pure utilitarian generalized overtaking criterion, say $R^{p u}$, generated by the pure utilitarian sequence $\left(R_{m}^{p u}\right)_{m=2}^{\infty}$.

Basu and Mitra (2003b) and Asheim and Tungodden (2004) propose alternative pure utilitarian criteria. They are formulated to compare streams of generational aggregated utility streams and give precedence to the present and near futures generations. Reformulated in our framework, Basu and Mitra (2003b) pure utilitarian SWQ, say $R^{P U}$, is defined by:

$$
\begin{aligned}
\forall u, v & \in \mathbb{R}^{\mathbb{N}}, u R^{P U} v \text { if and only if, for some } M \subset \mathbb{N}, \\
\sum_{i \in M} u_{i} & \geq \sum_{i \in M} v_{i} \text { and } u_{j} \geq v_{j}, \forall j \in \mathbb{N} \backslash M .
\end{aligned}
$$

By the above theorem, since $R^{P U}$ satisfies $\mathrm{SP}^{*}, \mathrm{FA}^{*}$ and extends $R_{2}^{p u}$, it is an extension of $R^{p u}$. Conversely, since $R^{p u}$ satisfies $\mathrm{SP}^{*}, \mathrm{FA}^{*}$ and is translatable 
(i.e. for any $u, v, w \in \mathbb{R}^{\mathbb{N}}, u R^{p u} v \Longleftrightarrow(u+w) R^{p u}(v+w)$ ), the argument ${ }^{4}$ of theorem 1 in Basu and Mitra (2003b) can be used to get that $R^{p u}$ is an extension of $R_{U}$. Asheim and Tungodden (2004) propose two alternative pure utilitarian criteria (a Catching Up and an Overtaking criterion) defined on infinite utility streams. These extensions are respectively characterized by $\mathrm{SP}^{*}$, FA*, a translation invariance condition and two alternative "Preference Continuity" conditions. Reformulated in our framework, both criteria would satisfy $R_{2}^{p u}$ so that they are extensions of $R^{p u}$ (but less partial).

These results suggest to use the following "simplified criterion":

Criterion 2 (simplified) Given a sequence of $S W Q s\left(R_{m}\right)_{m=2}^{\infty}$, a $S W Q R^{o}$ is $a$ simplified criterion if $\forall u, v \in \mathbb{R}^{\mathbb{N}}, u P^{o} v$ (resp. $u I^{o} v$ ) if and only if, for some $M \subset \mathbb{N}$ with $|M|=m, u_{M} P_{m} v_{M}$ and $u_{j} \geq v_{j}$ (resp. $u_{M} I_{m} v_{M}$ and $\left.u_{j}=v_{j}\right), \forall$ $j \in \mathbb{N} \backslash M$.

The following results shows that, when the sequence $\left(R_{m}\right)_{m=2}^{\infty}$ is proliferating, then a simplified criterion can be used equivalently to the generalised criterion generated by this sequence.

Theorem 4 Suppose $R^{o}$ is a generalised overtaking criterion generated by a proliferating sequence of $S W Q s\left(R_{m}\right)_{m=2}^{\infty}$, with $R_{2}$ satisfying $S P^{*}$ and $F A^{*}$ restricted to $\mathbb{R}^{2}$. Then $R^{o}$ is a simplified criterion.

Proof. Let $\hat{R}$ be a simplified criterion. That is: $\forall u, v \in \mathbb{R}^{\mathbb{N}}, u \hat{P} v$ (resp. $u \hat{I} v$ ) if and only if $u_{M} P_{m} v_{M}$ and $u_{j} \geq v_{j}$ (resp. $u_{M} I_{m} v_{M}$ and $u_{j}=v_{j}$ ), $\forall j \in \mathbb{N} \backslash M$, for some $M \subset \mathbb{N}$ with $|M|=m$. We want to show that, if $\hat{R}$ extends $R_{2}$, then $\forall u, v \in \mathbb{R}^{\mathbb{N}}, u \hat{R} v \Longleftrightarrow u R^{o} v$. Since $\hat{R}$ extends $R_{2}$ and, by lemma 3, satisfies SP* and FA*, we can apply theorem 3 and so get that $\hat{R}$ is an extension of $R^{\circ}$. Now, suppose $u, v \in \mathbb{R}^{\mathbb{N}}$ are such that for some $M \subset \mathbb{N}$ with $|M|=m, u_{M} P_{m} v_{M}$ and $u_{j} \geq v_{j}$ (resp. $u_{M} I_{m} v_{M}$ and $\left.u_{j}=v_{j}\right), \forall j \in \mathbb{N} \backslash M$. Since $R^{o}$ extends $R_{m}$, we have $u P^{o} v\left(\right.$ resp. $\left.u I^{o} v\right)$.

This theorem provides a much simpler characterization of the generalised overtaking criterion associated to a proliferating sequence. We have seen how it can be used in the pure utilitarian case. As another example it can be applied to the Leximin overtaking criterion, say $R^{L X}$ generated by the Leximin sequence $\left(R_{m}^{l x}\right)_{m=2}^{\infty}$. We have the following simplified criterion:

$$
\begin{aligned}
\forall u, v & \in \mathbb{R}^{\mathbb{N}}, u R^{L X} v \text { if and only if, for some } M \subset \mathbb{N},|M|=m, \\
u_{M} R_{m}^{l x} v_{M} \text { and } u_{j} & \geq v_{j}, \forall j \in \mathbb{N} \backslash M .
\end{aligned}
$$

By theorem 3, a SWQ $R$ defined on $\mathbb{R}^{\mathbb{N}}$ is an extension of $R^{L X}$ if and only if it satisfies $R_{2}^{l x}$ (and hence satisfies $\mathrm{SP}^{*}$ and $\mathrm{FA}^{*}$ ). As a result, $R^{L X}$ is equivalent to the Leximin criterion defined by Bossert, Sprumont and Suzumura (2004), any extension of which is characterized by $\mathrm{SP}^{*}, \mathrm{FA}^{*}$ and a two-person equity axiom, called Strict Equity Preference. This axiom is implied by Hammond Equity and SP* and, together with FA* implies the satisfaction of $R_{2}^{l x}$ (d'Aspremont, 1985, theorem 3.4.2). To recall, Hammond Equity, as a condition on a SWQ $R$, is: 
Hammond Equity $\left(H E^{*}\right)$. For any pair $\{i, j\} \subset N$, if $u$ and $v$ in $R^{\mathbb{N}}$ are such that $u_{j}=v_{j}$ for all $j \in \mathbb{N} \backslash\{i, j\}$, and $v_{i}<u_{i}<u_{j}<v_{j}$, then $u R v$.

Asheim and Tungodden (2004) propose two alternative Leximin criteria the extensions of which are characterized by $\mathrm{SP}^{*}, \mathrm{FA}^{*}$, Hammond Equity and, again, two alternative "Preference Continuity" conditions. In our framework, both criteria extend $R_{2}^{l x}$ so that they are extensions of $R^{L X}$ (but less partial).

There are many other examples of generalised overtaking criteria generated by proliferating sequences, since the proliferation property is a very common phenomenon among SWOs. To illustrate, we can show that pure utilitarianism can be generalised to a very large class of rules, the generalised pure utilitarian rules. The class is defined by:

Definition 5 (Generalised Pure $m$-Utlitarianism) The generalised pure utilitarian $S W O$ on $\mathbb{R}^{m}$, denoted $R_{m}^{g u}$, is such that: for any $u, v \in \mathbb{R}^{m}, u R_{m}^{g u} v$ if only if $\sum_{i=1}^{m} g\left(u_{i}\right) \geq \sum_{i=1}^{m} g\left(v_{i}\right)$, where the transformation $g$ is a continuous and increasing real-valued function defined on $\mathbb{R}$.

There are as many rules as there are transformations $g$. All these rules have the proliferation property and can therefore be used to define overtaking sequences in order to generate generalised overtaking criteria, to which the overtaking theorem can be applied. For any given transformation $g$, we call $\left(R_{m}^{g u}\right)_{m=2}^{\infty}$ the generalised pure utilitarian sequence. We then have:

Lemma 6 The generalised pure utilitarian sequence $\left(R_{m}^{g u}\right)_{m=2}^{\infty}$ is proliferating.

Proof. The proof is similar to the one for pure utilitarianism. Suppose a SWQ $R$ extends $R_{2}^{g u}, R_{3}^{g u}, \ldots$, and $R_{m}^{g u}$, we want to show that it extends $R_{m+1}^{g u}$. Again for simplicity, take $M^{\prime}=\{1,2, \ldots, m\} \cup\{m+1\}$, and any $u, v \in \mathbb{R}^{\mathbb{N}}$ such that $u_{i} \geq v_{i}, \forall i \in \mathbb{N} \backslash M^{\prime}$. Choose $w \in \mathbb{R}^{\mathbb{N}}$ such that $w_{i}=u_{i}$ for $1 \neq i \neq m+1$,

$$
g\left(w_{1}\right)+g\left(w_{m+1}\right)=g\left(u_{1}\right)+g\left(u_{m+1}\right),
$$

and $g\left(w_{m+1}\right)=g\left(v_{m+1}\right)$. Since $R$ extends $R_{2}^{g u}$, we have $w I u$. Also, since $R$ extends $R_{m}^{g u}$, we get

$$
\sum_{i=1}^{m+1} g\left(u_{i}\right)=\sum_{i=1}^{m+1} g\left(w_{i}\right)>\sum_{i=1}^{m+1} g\left(v_{i}\right) \Longrightarrow \sum_{i=1}^{m} g\left(w_{i}\right)>\sum_{i=1}^{m} g\left(v_{i}\right) \Longrightarrow w P v \Longrightarrow u P v
$$

and $u I v$ if the inequalities are replaced by equalities.

This class is characterized in Blackorby, Bossert and Donaldson (2002) in the finite, and in the variable, population case (for variants see Fleming, 1952, and Debreu, 1960).

The proliferating sequence $\left(R_{m}^{g u}\right)_{m=2}^{\infty}$ can be used to characterise a generalised pure utilitarian criterion $R^{g}$ for any transformation $g$, defined as a simplified criterion:

$$
\begin{aligned}
\forall u, v & \in \mathbb{R}^{\mathbb{N}}, u R^{g} v \text { if and only if, for some } M \subset \mathbb{N}, \\
\sum_{i \in M} g\left(u_{i}\right) & \geq \sum_{i \in M} g\left(v_{i}\right), \text { and } u_{j} \geq v_{j}, \forall j \in \mathbb{N} \backslash M .
\end{aligned}
$$


There are as many criteria as there are specifications of the transformation $g$, and these specifications depend on the additional conditions one wishes to impose. Various axiomatized specifications have been derived in the literature (see Blackorby, Bossert and Donaldson, 2002). We shall not review them here.

\section{Conclusion}

The characterisations we have derived for various SWOs over the infinite individual welfare evaluation space, all rely on a general overtaking criterion, and its simplified version, generated by some anonymous and strongly efficient SWQ that has the proliferating property. This property leads to the definition of an associated proliferating sequence the first element of which (satisfying strong Pareto and anonymity) plays the decisive role in the characterisation. This first element can sometimes be interpreted as an equity axiom. This is notably the case for the 2-leximin (the first element in the leximin proliferating sequence). But, this first element in the sequence can also be replaced by a set of other axioms that are known to be equivalent (from characterisations derived in the finite case). These characterisations, though, very often involve an "invariance" condition, restricting the measurability and interpersonal comparability properties of individual evaluation profiles. Not all invariance conditions can be admissible. For example, already in the finite case, FA* and noncomparability, in the sense of invariance with respect to individual increasing, or simply positive affine, transformations, imply universal social indifference (excluding SP*). Such results are reviewed in d'Aspremont and Gevers (2002). The extension of an invariance condition to the infinite-horizon case is even more delicate. However, some characterisations do work as the results of Asheim and Tungodden (2004) and of Basu and Mitra (2003b), mentioned above, do show. This line of investigation should be pursued.

Here, the overtaking theorem and its applications have been presented in order to stress an important consequence of sticking to welfarism in choosing criteria for intergenerational justice: adopting a criterion in evaluating the welfare of the present generation (or a subgroup) forces to use the same criterion for any subset of subsequent generations. This can be viewed as a very restrictive consequence, since it means that the moral value judgments of the first generation have to be imposed to all the subsequent ones. But it can also be seen, more positively, as a "time consistency" property. If the present generation solves, in some specific way, the social welfare evaluation problem, taking into consideration the social welfare evaluation of all future generations, this solution will be consistent with the solutions that future "present generations" should advocate. This is analogous to Rawls' conception (1971, p. 287) that looks at the problem "from the standpoint of the original position", where "the parties do not know to which generation they belong." Each generation solving the same problem (behind the veil of ignorance), and expecting the same solution being adopted by the other generations, has in fact correct expectations. 


\section{NOTES}

${ }^{*}$ I thank the participants to the IEA Roundtable Meeting on Intergenerational Equity, Hakone, Japan, 10-12 March 2005, and in particular Geir Asheim and Walter Bossert, for their fruitful comments and suggestions. Financial support from the Belgian Science Policy Office (CLIMNEG 2 and IAP programmes) is gratefully acknowledged.

${ }^{1}$ My dear friend and co-author Louis Gevers died in September 2004. His collaboration would have greatly improved the present work.

${ }^{2}$ See e.g. d'Aspremont and Gevers (1977), Sen (1977), d'Aspremont (1985) and Blackorby, Bossert and Donaldson (2002).

${ }^{3}$ The property is mentioned by Sen (1977, fn 26$)$ as suggested by Hammond as a step to derive the same property for Leximin. For a proof, see Hammond (1979).

${ }^{4}$ Basu and Mitra (2003b) assume that utility streams belong to $[0,1]^{\mathbb{N}}$, but the argument in their theorem 1 can be readily adapted.

\section{REFERENCES}

Arrow, K. J. (1951, 2d ed.1963), Social Choice and Individual Values, Wiley, New York.

Asheim, G. B. and Tungodden, B. (2004), "Resolving distributional conflicts between generations," Economic Theory 24, 221-230.

d'Aspremont, C. (1985), "Axioms for Social Welfare Orderings," in: Hurwicz, L., Schmeidler, D. and Sonnenschein, H. (eds.), Social Goals and Social Organizations: Essays in Memory of Elisha Pazner, Cambridge University Press, Cambridge, 19-76.

d'Aspremont, C. and Gevers, L. (1977), "Equity and the Informational Basis of Collective Choice," Review of Economic Studies 44, 199-209.

d'Aspremont, C. and Gevers, L. (2002), "Social Welfare Functionals and Interpersonal Comparability", in: Arrow, K., Sen A. and Suzumura K. (eds. ), Handbook of Social Choice and Welfare, vol.I, Elsevier, Amsterdam, 459-541.

Atsumi, H. (1965), "Neoclassical growth and the efficient program of capital accumulation," Review of Economic Studies 32, 127-136.

Basu, K. and Mitra, T. (2003a), "Aggregating Infinite Utility Streams with Intergenerational Equity: the Impossibility of Being Paretian," Econometrica 71, 1557-1563.

Basu, K. and Mitra, T. (2003b), "Utilitarianism for infinite utility streams: a new welfare criterion and its axiomatic characterisation," Working Paper \#0305, Department of Economics, Cornell University.

Blackorby, C., Bossert W. and Donaldson, D. (2002), "Utilitarianism and the Theory of Justice," in: Arrow, K., Sen A. and Suzumura K. (eds. ), Handbook of Social Choice and Welfare, vol.I, Elsevier, Amsterdam, 543-596.

Bossert, W., Sprumont Y. and Suzumura K. (2004), "The Possibility of Ordering Infinite Utility Streams," Cahier 12-2004, CIREQ.

Chakravarty, S. (1962), "The Existence of an Optimum Savings Program." Econometrica 30, 178-187.

Debreu, G. (1960), "Topological Methods in Cardinal Utility Theory," in: 
Arrow, K. J., Karlin, S., and Suppes, P. (eds.), Mathematical Methods in the Social Sciences, Stanford University Press, Stanford, 16-26.

Diamond, P. (1965), "The Evaluation of Infinite Utility Streams," Econometrica 33, 170-177.

Dhillon, A. (1998), "Extended Pareto Rules and Relative Utilitarianism," Social Choice and Welfare, 15, 521-542.

Ebert, U. (1988), "Measurement of Inequality: an Attempt at Unification and Generalization," Social Choice and Welfare 5, 147-169.

Fleming, M. (1952), "A Cardinal Concept of Welfare," Quarterly Journal of Economics 66, 366-384.

Fleurbaey, M. and Michel, P. (2003), "Intertemporal Equity and the Extension of the Ramsey Criterion," Journal of Mathematical Economics 39, 777-802.

Gevers, L. (1979), "On Interpersonal Comparability and Social Welfare Orderings," Econometrica 47, 75-89.

Hammond, P.J. (1976), "Equity, Arrow's Conditions, and Rawls' Difference Principle," Econometrica 44, 793-804.

Hammond, P.J. (1979), "Equity in Two Person Situations: Some Consequences," Econometrica 47, 1127-1135.

Kolm, S.C. (1972), Justice et Equite, CNRS, Paris.

Koopmans, T. C. (1960), "Stationary Ordinal Utility and Impatience," Econometrica 28, 287-309.

Lauwers L. (1998), "Intertemporal Objective Functions. Strong Pareto versus Anonymity," Mathematical Social Sciences 35, 37-55.

Lauwers, L. and Van Liedekerke, L. (1995), "Ultraproducts and Aggregation," Journal of Mathematical Economics 24, 217-237.

Mongin, P. and d'Aspremont, C. (1998), "Utility Theory and Ethics," in: Barberà S., Hammond P. and Seidl C. (eds.), Handbook of Utility Theory, vol. 1: Principles, Kluwer, Dordrecht, 371-481.

Ramsey, F. (1928), "A Mathematical Theory of Savings," Economic Journal $38,543-559$. Mass.

Rawls, J. (1971), A Theory of Justice, Harvard University Press, Cambridge,

Sen, A. K. (1970), Collective Choice and Social Welfare, Holden-Day, San Francisco.

Sen, A. K. (1976), "Welfare Inequalities and Rawlsian Axiomatics," Theory and Decision 7, 243-262.

Sen, A. K. (1977), "On Weights and Measures: Informational Constraints in Social Welfare Analysis," Econometrica 45, 1539-1572.

Suppes, P. (1966), "Some Formal Models of Grading Principles," Synthese 6, 284-306.

Svensson, L-G. (1980), "Equity among Generations," Econometrica 48, 12511256.

Szpilrajn, E. (1930), "Sur l'extension de l'ordre partiel," Fundamenta Mathematicae 16, 386-389.

Weymark, J.A. (1981), "Generalized Gini inequality indices," Mathematical Social Sciences 1, 409-430. 
von Weizsäcker, C. C. (1965), "Existence of Optimal Programmes of Accumulation for an Infinite Time Horizon," Review of Economic Studies 32, 85-104. 
Département des Sciences Économiques de l'Université catholique de Louvain

Institut de Recherches Économiques et Sociales

Place Montesquieu, 3

1348 Louvain-la-Neuve, Belgique 